\title{
Serological validation of an alveolar echinococcosis rat model with a single hepatic lesion
}

\author{
Masamichi YAMASHITA ${ }^{1)}$, Tomohiro IMAGAWA ${ }^{1)^{*}}$, Yasuhito SAKO ${ }^{2)}$, \\ Munehiro OKAMOTO ${ }^{3)}$, Tetsuya YANAGIDA ${ }^{4)}$, Yoshiharu OKAMOTO5), \\ Takeshi TSUKA ${ }^{1)}$, Tomohiro OSAKI ${ }^{5)}$ and Akira ITO ${ }^{2)}$ \\ 1)Department of Veterinary Diagnostic Imaging, School of Veterinary Medicine, Faculty of Agriculture, Tottori \\ University, Tottori 680-8553, Japan \\ ${ }^{2)}$ Department of Parasitology, Asahikawa Medical University, Asahikawa 078-8510, Japan \\ ${ }^{3)}$ Center for Human Evolution Modeling Research, Primate Research Institute, Kyoto University, Inuyama, Aichi \\ 484-8506, Japan \\ 4)Department of Parasitology, Joint Faculty of Veterinary Medicine, Yamaguchi University, Yamaguchi 753-8511, \\ Japan \\ ${ }^{5)}$ Department of Veterinary Surgery, School of Veterinary Medicine, Faculty of Agriculture, Tottori University, \\ Tottori 680-8553, Japan
}

J. Vet. Med. Sci.

79(2): 308-313, 2017

doi: 10.1292/jvms.16-0513

Received: 4 October 2016

Accepted: 12 November 2016

Published online in J-STAGE:

27 November 2016
ABSTRACT. Serology is important for the diagnosis and follow-up of human alveolar echinococcosis (AE). However, patient conditions are highly variable among those with $A E$, and antibody responses in serological follow-up have not been well-defined. We recently described a new $A E$ rat model established by implantation of small $A E$ tissue into a single arbitrary location in the liver; no metastasis and dissemination were observed. In the present study, we examined the serological characteristics in our rat model before and after surgical treatment. The results showed that antibody responses against crude antigens were increased at one month after transplantation and similar to those of other model animals. For the antigen Em18, antibody responses were slower in our rat model than in other animal models. After surgical resection, changes in antibody responses against Em18 were similar to those observed in human patients with AE. Because of the slow growth of lesions, establishment of a single hepatic lesion and patterns of antibody responses, our rat model may be useful for clarifying follow-up serodiagnoses in human $A E$ and determining the mechanisms of multi-organ involvement by primary infection with oncospheres rather than metastasis.

KEY WORDS: Echinococcus multilocularis, Em18, rat model, serology

Human alveolar echinococcosis (AE) is caused by the accidental ingestion of eggs of the fox tapeworm, Echinococcus multilocularis. AE is a typically neglected zoonotic disease occurring in the northern hemisphere. Recent reports have indicated that E. multilocularis infection is spreading among wild animals in Europe, Asia and North America; the increased incidence of AE is becoming a public health concern, in both developing and developed countries, because of the presence of foxes in urban areas in developed countries in Europe, Japan and Canada [4, 5, 14]. Human AE is characterized by hepatic malignant tumors, and if appropriate treatment is not provided, the patient may experience liver failure and even death [20, 22].

In human $\mathrm{AE}$, abdominal imaging to detect occupational lesions in the liver is essential, followed by reliable specific serology, as recommended by the World Health Organization [3, 22]. Serology using ezrin-radixin-moesin-like protein and EM10, EMII/3, EM4 or Em18 has been shown to be useful for serological differentiation of hepatic malignant tumors in patients with AE, hepatic cancer or other diseases and for the monitoring of AE cases after surgical and medical treatments [10]. In patients with AE, followup serology can vary widely according to patient condition, PNM stage, age, duration of infection, lesion size, and presence or absence of metastasis [7, 16, 27]. To validate the follow-up serodiagnosis, experimental animal models with lesions of uniform size and location as well as similar durations of infection are urgently needed.

Recently, we developed an AE model in which an alveolar hydatid vesicle was directly embedded into the liver of rats [31]. This model differs from previous animal models generated by oral inoculation with eggs of E. multilocularis and by infusion

*Correspondence to: Imagawa, T., Department of Veterinary Diagnostic Imaging, School of Veterinary Medicine, Faculty of Agriculture, Tottori University, Tottori 680-8553, Japan. e-mail: imagawat@muses.tottori-u.ac.jp

(02017 The Japanese Society of Veterinary Science

This is an open-access article distributed under the terms of the Creative Commons Attribution Non-Commercial No Derivatives (by-ncnd) License <http://creativecommons.org/licenses/by-nc-nd/4.0/>. 
of a homogenate of alveolar hydatid vesicle $[13,17,18]$, because the AE lesion is localized at an arbitrary point in the liver. Furthermore, recent studies re-evaluated the potential for the metastasis of AE lesions [2, 12]. Confirmation of disseminated AE lesions in this new model may support the occurrence of metastasis. Moreover, certain lesions may be completely resected, because of their localization. Thus, our AE model may be useful for observing host immune responses before and after treatment.

To examine the immune response in our rat model, we used two antigens (crude antigens of larval metacestode and recombinant Em18 [RecEm18] antigen). Em18, which can be either native or recombinant, has been shown to be highly useful and reliable for the differential diagnosis of human AE with approximately $90-100 \%$ specificity $[22,28]$, whereas crude antigens, which include highly cross-reactive nonspecific components, are expected to be useful for monitoring of the infection itself under experimental conditions. Although crude antigens are not used for differential diagnosis, they may be useful for preliminary screening [30]. Furthermore, the antibody levels generated in response to Em18 were found to be dramatically decreased after curative surgical resection of the whole lesion [27] and chemotherapy [7]. Thus, these antigens were used for follow-up after surgical treatment of AE.

In this study, we examined serological characteristics in our rat model and compared these characteristics with the features of human cases of AE and other model animals inoculated orally with eggs and injected with homogenates of alveolar hydatid vesicles. Our aim was to clarify whether our rat model could be used as an AE model for observing serological findings.

\section{MATERIALS AND METHODS}

\section{Model animals}

All procedures were carried out in accordance with the experimental animal institutional guidelines at Tottori University. The model animal was established as described previously [31]. Female Sprague-Dawley rats (4-5 weeks old; SLC; Hamamatsu, Japan; $n=9$ ) were used in this study. The rats were acclimated to an air-conditioned animal room for infected experimental animals (containment room, biosafety level 2) in the Experimental Animal Facility at Tottori University (lights on from 7 a.m. to 7 p.m.; and temperature, $25 \pm 3^{\circ} \mathrm{C}$ ). All animals had access to food and water ad libitum. Metacestodes of E. multilocularis were isolated from Myodes rufocanus bedfordiae in Ebetsu, Hokkaido, and passaged 10 times in BALB/c mice at Asahikawa Medical University. The metacestodes were divided into small pieces, and tiny vesicles were originally isolated under a stereomicroscope. Vesicles measuring 1.0-2.0 $\mathrm{mm}$ in diameter were inserted into the livers of six rats and covered with a gauze-type absorbable hemostat (Surgicel; Ethicon, Somerville, NJ, U.S.A.). Three control rats were treated in the same manner, but without transplantation. Plasma was collected and used to calculate the cut-off value.

Observation by magnetic resonance imaging (MRI) and blood sampling: After transplantation, blood sampling and MRI were carried out once a month to evaluate serological changes and changes in the sizes of transplanted lesions, respectively. Blood samples were collected from the common jugular vein, mixed with heparin and centrifuged at $1,000 \times \mathrm{g}$ for $10 \mathrm{~min}$ at $4^{\circ} \mathrm{C}$ to collect the plasma. The plasma was stored at $-80^{\circ} \mathrm{C}$ until analysis by enzyme-linked immunosorbent assays (ELISAs).

\section{Resection of the AE lesion}

After confirmation that the AE lesion proliferated and increased the antibody titer against RecEm18 ( $>0.2$, based on ELISA absorbance), the AE lesion was resected at 366 days after transplantation. Under isoflurane anesthesia, the median line of the abdomen was cut, and the part of the liver containing the cysts was resected by ligation at the proximal part of the cysts. After ensuring that no AE metacestodes remained in the liver or other abdominal organs, the abdominal cavity was closed. After the operation, blood was collected on days 1, 3, 5, 7,21 and 60. At 60 days after lesion resection, all rats were sacrificed, and the abdominal and thoracic cavities were examined macroscopically.

\section{Antigen preparation}

Antigens were prepared as described previously [19, 24-26]. Crude E. multilocularis antigen extract was prepared from fresh whole alveolar hydatid vesicle tissue materials. E. multilocularis metacestode tissue was obtained from nonobese diabetic severe combined immunodeficiency mice infected by intraperitoneal passage of metacestodes [19]. Microvesicle and protoscolex suspensions were prepared by pressing metacestode tissue through a 300- $\mu$ m metal mesh with phosphate-buffered saline (PBS). The microvesicles and protoscoleces were washed 5-7 times with PBS and homogenized with three volumes of lysis buffer consisting of $20 \mathrm{mM}$ Tris- $\mathrm{HCl}$ (pH 7.4), $150 \mathrm{mM} \mathrm{NaCl}$ and 1.0\% 3-([3-cholamidopropyl] dimethylammonia)-1-propanesulfonic acid in the presence of peptidase inhibitors (protease inhibitor cocktail for mammalian tissues; Sigma-Aldrich, St. Louis, MO, U.S.A.). After one freeze-thaw cycle and centrifugation at $10,000 \times g$ for $30 \mathrm{~min}$ at $4{ }^{\circ} \mathrm{C}$, the supernatant was recovered and stored at $-80^{\circ} \mathrm{C}$ until use [26]. RecEm18 protein was extracted from Escherichia coli harboring a RecEm18-expressing plasmid [24]. The expressed RecEm18 was purified using a chitin column (New England Biolabs, Ipswich, MA, U.S.A.) according to the manufacturer's instructions. Protein concentrations were determined using a BSA Protein Assay Kit (Pierce, Rockford, IL, U.S.A.) $[24,25]$.

\section{ELISA protocol}

ELISA was carried out as described previously [26]. Briefly, crude antigen and RecEm18 antigen were used to coat microtiter plates (Nunc-Immuno Plate; Thermo Fisher Scientific Inc., Waltham, MA, U.S.A.) at a concentration of $100 \mathrm{ng} /$ well. The wells were blocked with $250 \mu \mathrm{l}$ blocking buffer ( $20 \mathrm{mM}$ Tris- $\mathrm{HCl}$ [pH 7.4], $150 \mathrm{mM} \mathrm{NaCl}$ and $1 \%$ casein) at $37^{\circ} \mathrm{C}$ for $1 \mathrm{hr}$. After washing the wells with PBS containing 0.1\% Tween 20 (PBST), $100 \mu l$ of plasma diluted 1:100 in blocking buffer was added 

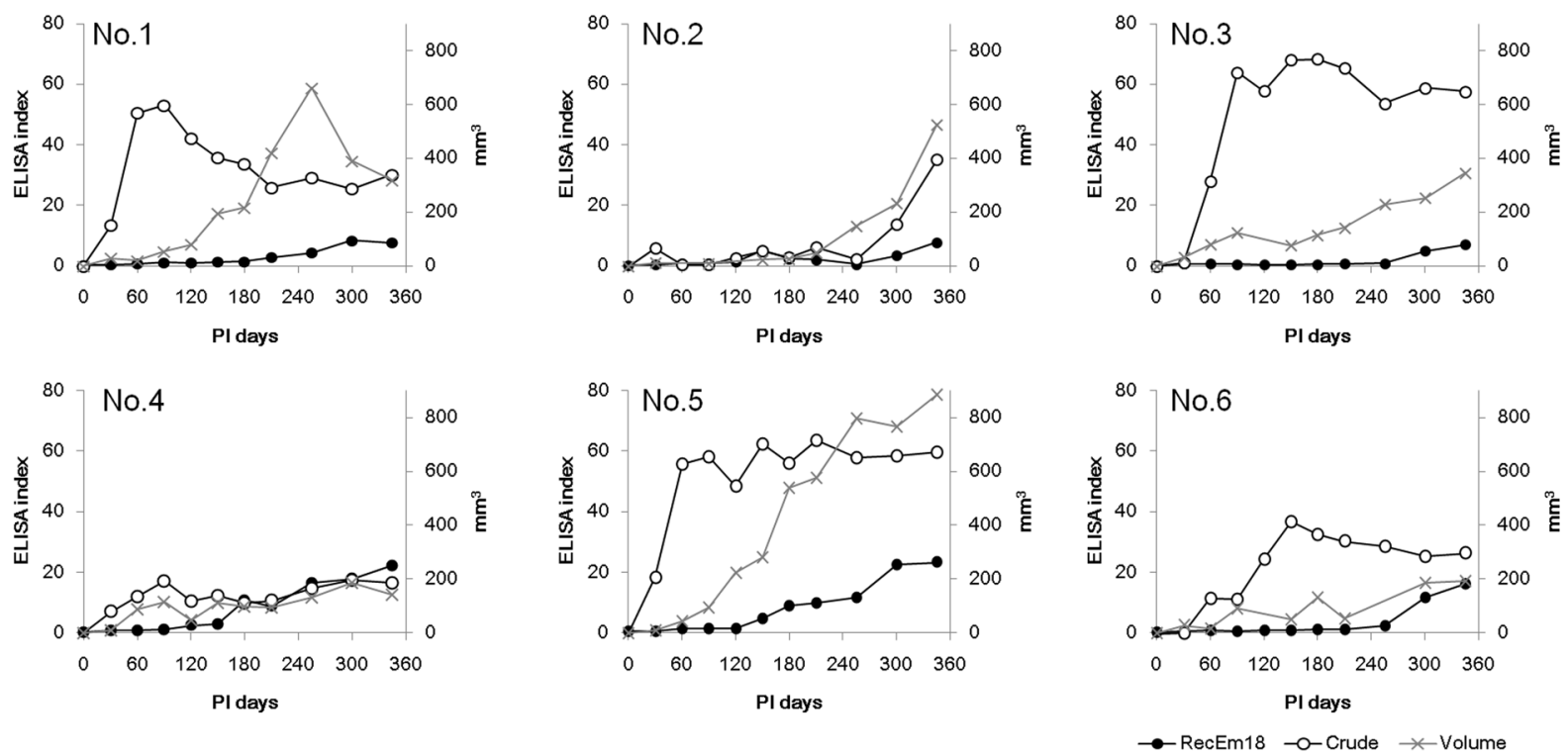

Fig. 1. Changes in antibody levels against RecEm18 (black circles) and crude antigen (white circles) and changes in the lesion area $(x)$ observed by MRI immediately after transplantation of vesicles into the livers of six rats and every 30 days until lesion resection. The antibody response for each antigen is shown as an ELISA index. The index of the individual plasma sample was calculated by dividing the sample's absorbance by the cutoff. The RecEm 18 cut-off value was 0.036 , and the crude antigen cut-off value was 0.014 . Cut-off $=1$ in the plot.

to each well, and the plates were then incubated at $37^{\circ} \mathrm{C}$ for $1 \mathrm{hr}$. The wells were washed four times with PBST, incubated with $100 \mu \mathrm{l}$ anti-rat IgG (Jackson ImmunoResearch, West Grove, PA, U.S.A.) at $37^{\circ} \mathrm{C}$ for $1 \mathrm{hr}$ and washed five times with PBST. After incubation with $400 \mu \mathrm{M}(100 \mu l)$ of 2,2'-azino-bis (3-ethylbenzothiazoline-6-sulfonic acid) diammonium salt (Sigma-Aldrich) for $30 \mathrm{~min}$ at room temperature, absorbance was measured at $405 \mathrm{~nm}$ with a reference wavelength of $490 \mathrm{~nm}$, as described previously [26]. The cut-off values for each antigen were defined as the average absorbance + standard deviation $\times 4$ of three control rats. The index of the individual plasma sample was calculated by dividing the sample's absorbance by the cutoff.

\section{$M R I$}

MRI was performed using a 0.3 T AIRIS Vento instrument (HITACHI Medico Inc., Tokyo, Japan) under isoflurane anesthesia. The following parameters were employed: field of view, $180 \mathrm{~mm}$; slice thickness, $2.5 \mathrm{~mm}$; and interval, $3.0 \mathrm{~mm}$. T1-weighted and T2-weighted images were obtained with repetition/echo times of 380/15 and 4,000/100 ms, respectively. Volumes of the AE lesions were calculated as (major axis $\times$ minor axis $\left.{ }^{2}\right) / 2$ for each slice.

\section{Histological examination}

The AE lesions in rat Nos. 2, 5 and 6 and the tissues in which the AE lesion exhibited suspected metastasis or dissemination were removed and fixed in neutral-buffered $10 \%$ formalin and then embedded in paraffin. Five-micrometer-thick sections of the lesions were stained with hematoxylin and eosin.

\section{RESULTS}

After transplantation, the AE lesions were observed by MRI. In all rats, the lesions were localized at a single point in the liver, and no metastasis of metacestodes was observed in the thoracic and abdominal cavities. Moreover, after necropsy, other AE lesions were not macroscopically observed in any rat.

Blood was sampled immediately before hydatid transplantation and at approximately monthly intervals thereafter. In nearly all individuals, approximately $1 \mathrm{~m} l$ blood was sampled at each blood draw, and we were able to measure the antibody titers for each antigen over time. Figure 1 shows the ELISA index against RecEm18 and the crude antigen, which was calculated based on absorbance using cut-off values established in control rats from immediately before transplantation until lesion resection. The antibody against the crude antigen increased in all individuals; antibody positivity was observed on day 30 after infection. The mean timing of antibody positivity in against RecEm18 was 120 days, and the antibody level continued to increase from the initial response until resection of the lesions.

The lesions were confirmed by MRI beginning 30 days after infection in all six rats, and growth of the AE lesion was observed (Fig. 1). Rapid lesion growth was observed from 60 to 150 days (average of $82 \pm 31$ days) after transplantation. The standard deviation was not large; however, the subsequent rate of lesion growth differed among individuals. Additionally, even at 360 days 
A

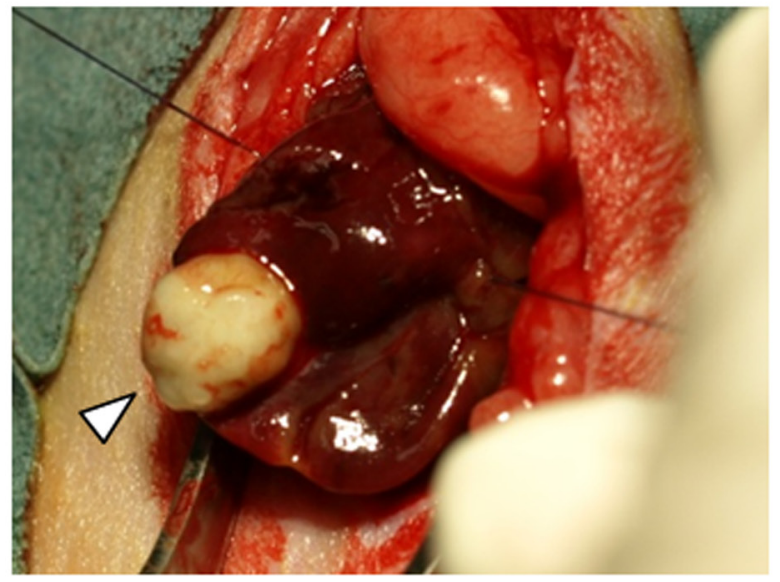

B

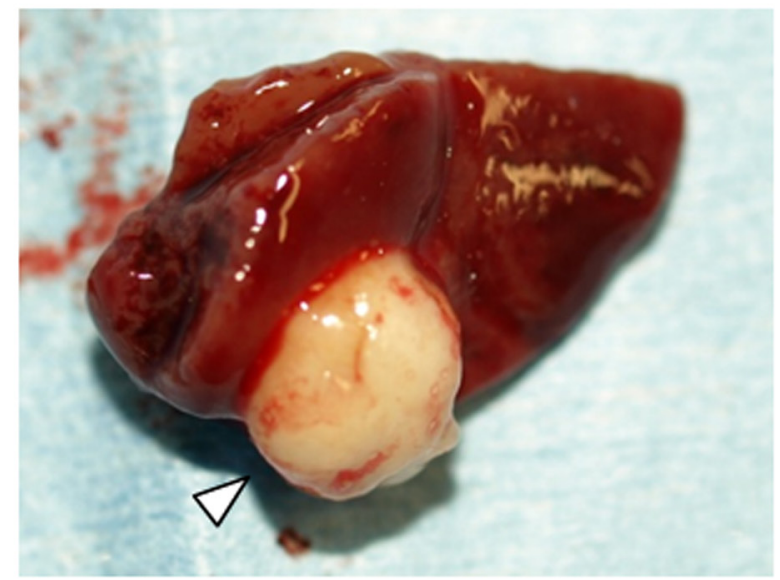

Fig. 2. (A) Resection of AE lesions. (B) Resected lesion and liver margin. The arrowhead indicates the AE lesion.
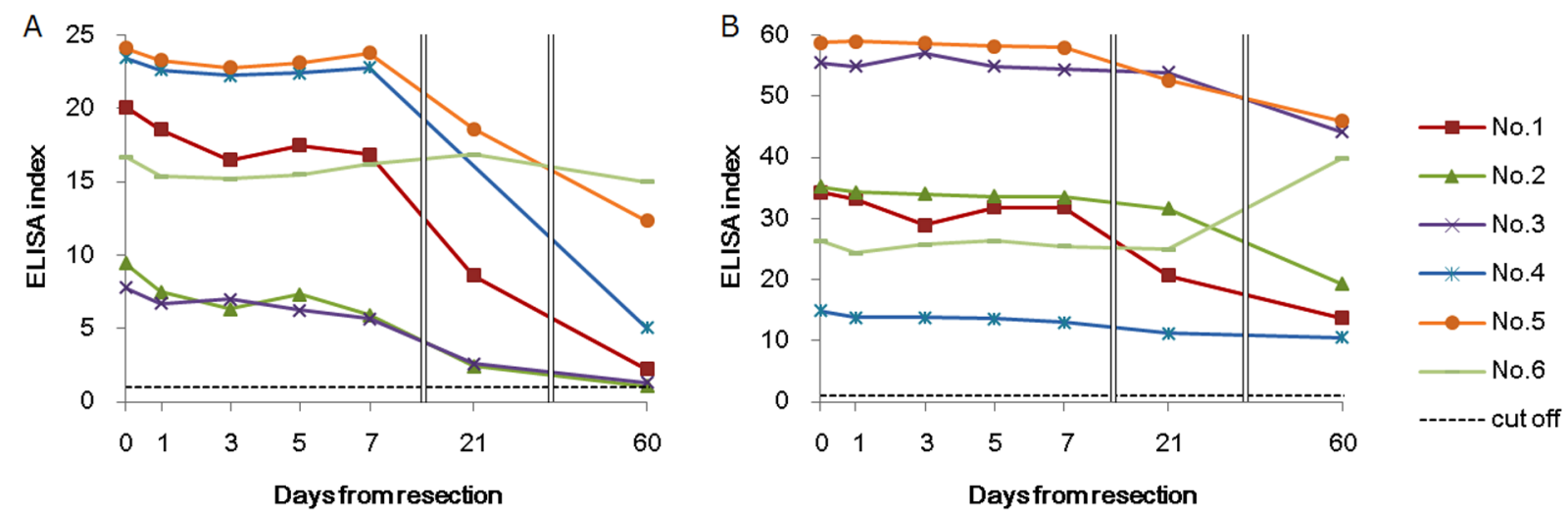

Fig. 3. Changes in antibody responses against RecEm18 (A) and crude antigen (B) after lesion resection. Blood was sampled on days $0,1,3,5,7,21$ and 60 after lesion resection to examine the antibody response. The antibody response for each antigen is shown as an ELISA index. The index of the individual plasma sample was calculated by dividing the sample's absorbance by the cutoff. The RecEm 18 cut-off value was 0.036 , and the crude antigen cut-off value was 0.014 . Cut-off $=1$ in the plot.

after transplantation, a lesion was only detected at one site in the liver.

Figure 2 shows one of the resected lesions. The focus was localized to the tail side of the left lateral lobe. For all rats, the focus could be completely removed by resecting half of the affected lobe. The resected lesion ranged from 9 to $15 \mathrm{~mm}$ in diameter. In the histological findings of AE lesions, protoscolices were not found, and germinal cells were poor. After completion of the experiment, we performed gross observations of the rat lungs, liver and abdominal cavity, and the tissues in which AE lesion showed suspected metastasis or dissemination were evaluated histologically, but no metastasis or dissemination was detected.

We acquired blood samples after treatment every other day $(0.5 \mathrm{~m} l)$ for 1 week after resection. The antibody against Em 18 decreased in all rats after resection. By day 21 , the antibody level was reduced by $50 \%$ in three rats, while by day 60 , the antibody level was reduced by $50 \%$ in five rats; however, the antibody response did not completely disappear in any individual (Fig. 3). The antibody against the crude antigen was decreased after resection; however, the reduction was more gradual than that of the Em18 antibody levels. Similarly to the antibody response against Em18, the antibody did not completely disappear in any individual within 60 days. The rate of change for both antibody levels against Em18 and crude antigen was similar in each individual after lesion resection; however, the ELISA index (estimated at the time of lesion resection) exhibited substantial variations among individuals. In addition, in rat No. 6, the reduction in the antibody response to Em18 was slow and minor, while the antibody response to the crude antigen increased postoperatively.

\section{DISCUSSION}

After vesicle transplantation, we observed antibody responses and lesion sizes for an extended duration. Based on MRI analysis, 
the lesions were localized to one part of the liver during the entire study period. Lesion growth was observed at 81 days after transplantation, and lesion size increased until 360 days. No lesions occupied the entire liver or other tissues or organs. In mouse models in which eggs were ingested or homogenates were injected into the liver or portal vein, lesions increased substantially and occupied the whole liver within 20 weeks [11, 13, 17, 18]. Moreover, metastasis or multiple lesions are formed in egg and homogenate models, in contrast to the results of the present model [13, 17, 18]. Based on our results and those of previous studies, we concluded that lesion growth in our model was much slower than that in other models. In human AE, there are no suitable tools for detecting early hepatic stage $\mathrm{AE}$ with lesions less than 1 or $3 \mathrm{~cm}$ in size, except for needle biopsy [2]. Additionally, the latent period is very long; it can take several years after infection for patients to be diagnosed [15, 29]. The slow growth and hepatic localization of lesions observed in this model were similar to those in human AE. Using the present rat model, immune responses and lesion sizes (with the absence of metastasis) were followed for over 1 year. Thus, the present model may be suitable for longterm observations, including observation of metastasis.

In the present study, ELISA indices against crude and RecEm18 antigens showed high variations among individual rats. However, we validated the changes in lesion sizes and antibody responses against crude and Em18 antigens in each rat. After transplantation of AE vesicles, antibody responses against crude antigens were observed on day 30, and those against Em18 were observed on day 120 on average in six rats. Human AE is rarely diagnosed during the early hepatic stage, and the time course of the immune response is unclear. Nearly all early AE cases are accidentally confirmed based on misdiagnosis of metastasis of rectal or stomach cancer [2]. In two experimental mouse models of oral inoculation with eggs, antibody responses against crude antigens were initially detected at 4 and 9 weeks $[17,18]$, and in an experiment involving homogenate injection, antibody responses were detected at 5-10 weeks [29]. Antibody responses against II/3 antigen, a source of Em18, were detected at 4 weeks after egg inoculation in a mouse model [17]. When comparing the current results with these previous reports, the timing of antibody responses against crude antigens was similar, but the response to Em18 occurred later than that in a previous report of egg inoculation. These findings suggest that the changes in antibody responses against Em18 in the present model may be similar to those observed in human AE. In the present model, the antibody response against Em18 antigen was increased; however, the AE lesions had no protoscolices. Although the source of Em18 antigen is protoscolices, Em10, a source of Em18, is also expressed in germinal epithelium of brood capsules [6]. Protoscolices are rarely formed in the human host [22], and the histological finding of the present rat model was similar to human AE.

Using our animal model, we generated a lesion in an arbitrary location in the liver, facilitating complete resection. Furthermore, frequent blood sampling after resection was unproblematic in rats with no clinical symptoms. The antibody responses against crude antigens showed minimal reduction until 60 days after the resection of AE lesions. In contrast, those against Em18 were decreased by approximately $50 \%$ in five of six rats until 60 days. In a previous study of human patients with AE, the antibody responses against Em18 and crude antigens were decreased after surgical treatment when observed at 6-month intervals [7, 27]. Moreover, the indices of crude antigens changed more slowly than those of tests using single recombinant or other affinity-purified antigens [27]. Recent studies reported two cases of human AE in which antibody responses against RecEm18 began declining within a few days after resection and were negative within 6 months $[1,8]$. In animal models in which homogenates were injected into the liver, the antibody responses against crude antigens were rarely decreased at 35 weeks after resection of the AE lesion [29]. Reduction of the antibody response against crude antigen in the present study was similar to that in previous human cases rather than in animal models. Moreover, the decreased antibody responses against Em18 in the present study were more rapid than those previously observed in human cases at 6-month intervals [7,27], but were similar to or slightly slower than those in other studies of human cases $[1,8]$. Based on these findings, the changes in antibody responses in our current model may resemble those of human AE. In one rat, the levels of antibodies against crude antigen and RecEm18 were not decreased. We evaluated the liver, lungs and intraperitoneal tissues using MRI and gross necropsy to ensure that there was no remaining parasite tissue; however, it is possible that undetectable micrometastases or parasite tissue was overlooked.

As described above, multi-organ AE cases involving the lung and brain have been explained by metastasis [21, 22]. However, our study provided no evidence of metastasis. Furthermore, recent studies by Aoki et al. [2] and Kvascevicius et al. [12] in AE cases and by Sadjjadi et al. [23] in cases of cystic echinococcosis (CE) suggested that the occurrence of multi-organ echinococcosis, either AE or CE, may be due to invasion by multiple oncospheres in all primary lesions in the liver, lung and brain. This hypothesis was also supported by the findings of Ito et al. [9]. Thus, our animal model is expected to facilitate additional studies of metastastic potential.

In summary, we elucidated the serological characteristics of our rat model. Our findings demonstrated that the rat model resembled the pathological features of AE lesions with slow and localized growth in the liver and serological responses of human $\mathrm{AE}$ to a greater extent than other previously reported animal models. Furthermore, our model permitted the long-term monitoring of AE lesions.

\section{REFERENCES}

1. Akabane, H., Nakano, S., Inagaki, M., Yanagida, N., Shoumura, H., Kudo, T., Shonaka, T., Orimo, T., Oikawa, F., Aiyama, T., Shibaki, T., Sako, Y., Itoh, S. and Ito, A. 2012. Evaluation of a long term follows up by imaging and serology on a hepatic alveolar hydatidosis at Asahikawa Kousei Hospital. Hokkaido Noson Igaku 44: 1-7 (in Japanese).

2. Aoki, T., Hagiwara, M., Yabuki, H. and Ito, A. 2015. Unique MRI findings for differentiation of an early stage of hepatic alveolar echinococcosis. BMJ Case Rep. 2015: bcr2014208123. [CrossRef] [Medline] 
3. Brunetti, E., Kern, P., Vuitton D. A., Writing Panel for the WHO-IWGE 2010. Expert consensus for the diagnosis and treatment of cystic and alveolar echinococcosis in humans. Acta Trop. 114: 1-16. [Medline] [CrossRef]

4. Davidson, R. K., Romig, T., Jenkins, E., Tryland, M. and Robertson, L. J. 2012. The impact of globalisation on the distribution of Echinococcus multilocularis. Trends Parasitol. 28: 239-247. [Medline] [CrossRef]

5. Deplazes, P., Hegglin, D., Gloor, S. and Romig, T. 2004. Wilderness in the city: the urbanization of Echinococcus multilocularis. Trends Parasitol. 20: 77-84. [Medline] [CrossRef]

6. Frosch, P. M., Frosch, M., Pfister, T., Schaad, V. and Bitter-Suermann, D. 1991. Cloning and characterisation of an immunodominant major surface antigen of Echinococcus multilocularis. Mol. Biochem. Parasitol. 48: 121-130. [Medline] [CrossRef]

7. Ishikawa, Y., Sako, Y., Itoh, S., Ohtake, T., Kohgo, Y., Matsuno, T., Ohsaki, Y., Miyokawa, N., Nakao, M., Nakaya, K. and Ito, A. 2009. Serological monitoring of progression of alveolar echinococcosis with multiorgan involvement by use of recombinant Em18. J. Clin. Microbiol. 47: 3191-3196. [Medline] [CrossRef]

8. Ito, A. 2013. Nothing is perfect! Trouble-shooting in immunological and molecular studies of cestode infections. Parasitology 140: 1551-1565. [Medline] [CrossRef]

9. Ito, A., Nakao, M. and Sako, Y. 2007. Echinococcosis: serological detection of patients and molecular identification of parasites. Future Microbiol. 2: 439-449. [Medline] [CrossRef]

10. Ito, A., Nakao, M., Lavikainen, A. and Hoberg, E. 2016. Cystic echinococcosis: Future perspectives of molecular epidemiology. Acta Trop. S0001706X(16)30242-X (in press). [Medline]

11. Ito, A., Kanazawa, T., Nakao, M., Sako, Y., Ishikawa, Y. and Nakaya, K. 2001. Comparison of the antigenicity of protoscoleces and microvesicles of Echinococcus multilocularis prepared from rats. J. Helminthol. 75: 355-358. [Medline]

12. Kvascevicius, R., Lapteva, O., Al, A. O., Audronyte, E., Neverauskiene, L., Kvasceviciene, E., Sokolovas, V., Strupas, K., Marcinkute, A., Deplazes, P. and Müllhaupt, B. 2016. Fatal liver and lung alveolar echinococcosis with newly developed neurologic symptoms due to the brain involvement. Surg. J. 2: e83-e88. [CrossRef]

13. Liance, M., Vuitton, D. A., Guerret-Stocker, S., Carbillet, J. P., Grimaud, J. A. and Houin, R. 1984. Experimental alveolar echinococcosis. Suitability of a murine model of intrahepatic infection by Echinococcus multilocularis for immunological studies. Experientia 40: 1436-1439. [Medline] [CrossRef]

14. Liccioli, S., Giraudoux, P., Deplazes, P. and Massolo, A. 2015. Wilderness in the 'city' revisited: different urbes shape transmission of Echinococcus multilocularis by altering predator and prey communities. Trends Parasitol. 31: 297-305. [Medline] [CrossRef]

15. Lightowlers, M. W., Mitchell, G. F. and Rickard, M. D. 1993. Immunology and molecular biology of parasitic infection. pp. 438-472. In: Cestodes. (Warren, K.S. and Agabian, N. eds.), Blackwell Scientific, Oxford.

16. Ma, L., Ito, A., Liu, Y. H., Wang, X. G., Yao, Y. Q., Yu, D. G. and Chen, Y. T. 1997. Alveolar echinococcosis: Em2plus-ELISA and Em18-western blots for follow-up after treatment with albendazole. Trans. R. Soc. Trop. Med. Hyg. 91: 476-478. [Medline] [CrossRef]

17. Matsumoto, J., Kouguchi, H., Oku, Y. and Yagi, K. 2010. Primary alveolar echinococcosis: course of larval development and antibody responses in intermediate host rodents with different genetic backgrounds after oral infection with eggs of Echinococcus multilocularis. Parasitol. Int. 59: 435-444. [Medline] [CrossRef]

18. Matsumoto, J., Yagi, K., Nonaka, N., Oku, Y. and Kamiya, M. 1998. Time-course of antibody response in mice against oral infection with eggs of Echinococcus multilocularis. Parasitology 116: 463-469. [Medline] [CrossRef]

19. Nakaya, K., Mamuti, W., Xiao, N., Sato, M. O., Wandra, T., Nakao, M., Sako, Y., Yamasaki, H., Ishikawa, Y., Craig, P. S., Schantz, P. M. and Ito, A. 2006. Usefulness of severe combined immunodeficiency (scid) and inbred mice for studies of cysticercosis and echinococcosis. Parasitol. Int. $\mathbf{5 5}$ Suppl: S91-S97. [Medline] [CrossRef]

20. Nunnari, G., Pinzone, M. R., Gruttadauria, S., Celesia, B. M., Madeddu, G., Malaguarnera, G., Pavone, P., Cappellani, A. and Cacopardo, B. 2012. Hepatic echinococcosis: clinical and therapeutic aspects. World J. Gastroenterol. 18: 1448-1458. [Medline] [CrossRef]

21. Ozdol, C., Yildirim, A. E., Daglioglu, E., Divanlioglu, D., Erdem, E. and Belen, D. 2011. Alveolar hydatid cyst mimicking cerebellar metastatic tumor. Surg. Neurol. Int. 2: 13. [Medline] [CrossRef]

22. Pawlowski, Z. S., Eckert, J., Vuitton, D. A., Ammann, R. W., Kern, P., Craig, P. S., Dar, K. F., De Rosa, F., Filice, C., Gottstein, B., Grimm, F., MacPherson, C. N. L., Sato, N., Todorov, T., Uchio, J., von Sinner, W. and Wen, H. 2001. Hydatidosis in humans: clinical aspects, diagnosis and treatment. pp. 20-71. In: WHO/OIE Manual on Hydatidosis in Humans and Animals: A Public Health Problem of Global Concern. (Eckert, J., Gemmell, M. A., Meslin, F. X. and Pawlowski, Z. S. eds.), World Organization for Animal Health, Paris.

23. Sadjjadi, S. M., Mikaeili, F., Karamian, M., Maraghi, S., Sadjjadi, F. S., Shariat-Torbaghan, S. and Kia, E. B. 2013. Evidence that the Echinococcus granulosus G6 genotype has an affinity for the brain in humans. Int. J. Parasitol. 43: 875-877. [Medline] [CrossRef]

24. Sako, Y., Fukuda, K., Kobayashi, Y. and Ito, A. 2009. Development of an immunochromatographic test to detect antibodies against recombinant Em18 for diagnosis of alveolar echinococcosis. J. Clin. Microbiol. 47: 252-254. [Medline] [CrossRef]

25. Sako, Y., Nakao, M., Nakaya, K., Yamasaki, H., Gottstein, B., Lightowers, M. W., Schantz, P. M. and Ito, A. 2002. Alveolar echinococcosis: characterization of diagnostic antigen Em18 and serological evaluation of recombinant Em18. J. Clin. Microbiol. 40: 2760-2765. [Medline] [CrossRef]

26. Sako, Y., Tappe, D., Fukuda, K., Kobayashi, Y., Itoh, S., Frosch, M., Grüner, B., Kern, P. and Ito, A. 2011. Immunochromatographic test with recombinant Em18 antigen for the follow-up study of alveolar echinococcosis. Clin. Vaccine Immunol. 18: 1302-1305. [Medline] [CrossRef]

27. Tappe, D., Frosch, M., Sako, Y., Itoh, S., Grüner, B., Reuter, S., Nakao, M., Ito, A. and Kern, P. 2009. Close relationship between clinical regression and specific serology in the follow-up of patients with alveolar echinococcosis in different clinical stages. Am. J. Trop. Med. Hyg. 80: $792-797$. [Medline]

28. Xiao, N., Mamuti, W., Yamasaki, H., Sako, Y., Nakao, M., Nakaya, K., Gottstein, B., Schantz, P. M., Lightowlers, M. W., Craig, P. S. and Ito, A. 2003. Evaluation of use of recombinant Em18 and affinity-purified Em 18 for serological differentiation of alveolar hydatidosis from cystic hydatidosis and other parasitic infections. J. Clin. Microbiol. 41: 3351-3353. [Medline] [CrossRef]

29. Yamano, K., Miyoshi, M., Goto, A. and Kawase, S. 2014. Time course of the antibody response in humans compared with rats experimentally infected with hepatic alveolar echinococcosis. J. Helminthol. 88: 24-31. [Medline] [CrossRef]

30. Yamano, K., Goto, A., Miyoshi, M., Furuya, K., Sawada, Y. and Sato, N. 2009. Diagnosis of alveolar echinococcosis using immunoblotting with plural low molecular weight antigens. J. Helminthol. 83: 57-61. [Medline] [CrossRef]

31. Yamashita, M., Imagawa, T., Nakaya, K., Sako, Y., Okamoto, Y., Tsuka, T., Osaki, T., Okamoto, M. and Ito, A. 2013. Echinococcus multilocularis: Single hepatic lesion experimentally established without metastasis in rats. Exp. Parasitol. 135: 320-324. [Medline] [CrossRef] 